This is a draft chapter. The final version will be available in Computational Legal Studies: The Promise and Challenge of Data-Driven Legal Research edited by Ryan Whalen, forthcoming 2019, Edward Elgar Publishing Ltd. The material cannot be used for any other purpose without further permission of the publisher, and is for private use only.

\title{
Is Legal Cognition Computational? (When Will DeepVehicle Replace Judge Hercules?)
}

\author{
Paul Gowder, University of Iowa
}

\begin{abstract}
Could we insert machine learning into the adjudicative process? This chapter considers the extent of the isomorphism between common-law reasoning from prior cases and machine learning reasoning from prior observations, as well as the normative considerations governing any such use. It ultimately concludes that we could use machine learning models to assist judges in reasoning about some questions of law, but only in the context of an ordinary legal process regulating both the use and the forms of such models. Ultimately, machine learning would be less likely to replace judicial reasoning and legal argument than to move it around.
\end{abstract}

Keywords: machine learning, adjudication, jurisprudence, disagreement, argumentation, legal reasoning

\section{The Self-Abnegating Judge}

Let us suppose that we could represent the facts of some legal dispute in some tabular form. As we shall shortly see, that assumption ignores some dicey initial problems, but grant it to me for just a moment. ${ }^{1}$

${ }^{1}$ In particular, I am deliberately assuming away the immense practical and conceptual problems that Pasquale and Cashwell (2018) have identified in existing efforts to use machine learning to come to legal judgments: the prior work to identify meaning in those facts, which Pasquale and Cashwell have identified as the core problem, has by assumption already been done. Later on in this chapter I will relax this assumption. I make no claim 
Since this is sort of a jurisprudence chapter, suppose the law is none other than "no vehicles in the park."2 Further suppose that we do not have a prior case "on all fours" with the instant problem - the defendant has brought some novel thing into the park, and the judge is uncertain about whether it ought to be classified as a vehicle. But we do have a tabular representation of every relevant fact about the thing that the defendant brought into the park, and a similar representation of the facts and result of every other of the (by assumption) very large number of cases about the vehicles in the park law.

Here's a segment of that table of prior cases, as we might imagine it:

\begin{tabular}{llll} 
Weight & Number of wheels & Engine & Vehicle? \\
\hline 5 & 3 & T & T \\
90 & 2 & T & F \\
10 & 8 & F & F
\end{tabular}

that this is a project that we're likely to be able to undertake in the immediate future. First of all, any useful machine learning model for adjudication would require lots of training data, which currently does not exist in any useful form for most areas of law. It would be extremely expensive to collect any such data-and it might be that the only way to do it would be to wait for for-profit companies in particularly lucrative areas of law to build predictive models for the use of litigators, and then find some way to capture them for public use. In short, this is a philosopher's thought experiment, not a practical set of recommendations for creating machine learning judges. That being said, the law has often been surprisingly amenable to methodological experimentation in the past; the conclusion to this chapter discusses some practical considerations for managing such experimentation in this context.

2 This chapter will not spend a lot of energy closely parsing the philosophy of law literature-I assume that the reader is broadly familiar with the ideas extant in conceptual jurisprudence. I don't really intend to contribute directly to either the jurisprudence literature or to the machine learning literature, but rather to put the two in conversation and see if anything interesting comes out. For a preliminary anchor in the former, allow me to here gesture at the notion that contemplating the role of machine learning in adjudication is most productive when we start from a view that more-or-less resembles Waldron's (2004) proceduralist reading of Dworkin. (For those who are not jurisprudence people, the no vehicles in the park example is probably the most important thought experiment in 20th century conceptual jurisprudence, and comes from Hart (1958), and Judge Hercules comes from Dworkin (1986).) On the machine learning side, my thanks to Stephen Tu for correcting some of my terminological errors. 
Let us make one more (sadly unrealistic) assumption: our judge (Judge Hercules, of course) is an expert in machine learning. So she reasons as follows: "I could try to use my best judgment to figure out whether the thing Defendant brought into the park is a vehicle. In order to do so, I'm going to have to find a rule from my knowledge of how prior cases came out. But computers can more cheaply and more comprehensively generalize the prior cases, without my biases and idiosyncrasies. So I should fit a machine learning model to all the prior vehicle in the park fact patterns, generate a prediction about the current facts, and rule accordingly." 3

Ought our judge do that? Ought the legislature mandate that she do so? Would the use of this model (let's call it DeepVehicle) represent a coherent conception of judicial judgment? That's what this chapter considers.

\section{Estimating Legal Truth as a Function}

So here's one story we could tell, which might lead us to approve of our judge's idea. Within some constraints that aren't relevant here, certain popular machine learning techniques (like a neural network) are known, mathematically, to be able to approximate any function (Lin, Tegmark, and Rolnick 2017, 1227-8). Judicial decision-making on questions of law (given an absence of dispute of material fact) seems to be something like a function-we can abstractly conceive of the judge as observing (with some error) an underlying legal rule that maps a vector of facts (from within the domain of all possible legally relevant facts) to some vector of legal statements including propositions like "the thing the defendant brought into the park counts as a vehicle."

Moreover, on many interpretations of the normative ideal of the rule of law, it's important that legal rules are determinate-or, rephrased, legal rules ought not to vary with irrelevant facts (facts not properly included in our notional vector) such as the identity of the judge (Gowder 2014a, 1069). That property of law is more-or-less equivalent to the property of mathematical functions that each input to a function maps to only one output. Furthermore, judges are institutionally constrained from throwing up their hands and saying "I don't know." In mathematical terms, that's more or less equivalent to the property of functions that they map everything in their domain to some output. So (Judge Hercules reasons) the obligation of a judge to find the exactly one legally correct outcome for a given vector of facts is equivalent to an unknown function called "the legal rule"; we ought to be able to find some machine learning model that approximates that rule.

Furthermore (Judge Hercules continues to reason), ordinary legal practice in common law systems requires judges to discover that legal rule from the prior cases. ${ }^{4}$ The process of

3 This is not wholly far-fetched: legal scholars have already begun to make efforts to predict judicial results using machine learning, although their approaches have not, thus far, been specifically limited to legally relevant facts (e.g. Aletras et. al. 2016, Katz et. al. 2017.).

${ }^{4}$ As should be clear, I'm assuming something like a common law jurisdiction where (even in the interpretation of a statute), a judge is formally bound to take into consideration the 
legal reasoning from those prior decisions is what first-year law students are taught as "following" and "distinguishing" precedent. We can easily interpret this process as analogous to the mathematical operation of finding a line (or plane, or hyperplane, depending on the dimension of the input fact-space) that best separates the points in space that belong to the various legal judgments-coming to the judgment that, for example, the rule that best takes into account the previous cases is that everything that has an engine, or weighs more than 50 pounds, is a vehicle.

I'll restate the previous paragraph a little more precisely. Here's how our data scientist judge might think about the activities of "following" and "distinguishing." To follow a case is just to assert that those facts of that case that mattered for its result are relevantly similar to the facts in the present case. We could represent the set of categories in which those facts fall, or, equivalently, the questions to which each fact is an answer, like "how many wheels does the alleged vehicle have?," as an n-dimensional space where each dimension corresponds to one of those questions. Then we can represent each prior case as some point in that space, and our rule for picking out what is and what is not a vehicle as some ndimensional hyperplane separating the half-space containing cases in which the thing defendant brought into the park was ruled a vehicle from the half-space where the thing defendant brought into the park was not ruled a vehicle. ${ }^{5}$ In that representation, to follow a case is to claim that the facts of the present case are on the same side of the plane as the case you're following; to distinguish a case is to say that it's on the opposite side.

Here's another direction from which to approach the same story. The tech industry routinely makes use of our labor to train machine learning models to reflect human judgment. When we answer a Google image "captcha" that asks us to identify, for example, every segment of an image reflecting a street sign, we're contributing a row of data to be used by artificial intelligence to identify those signs without our help, by aggregating the wisdom of millions of humans who have somehow figured out how to carry out that task. ${ }^{6}$ The goal is to enable the computer to come to the same answer that a human would.

Part of what makes this interesting, I contend, is that street signs are conventionally constructed: the answer to the question "is this a street sign" is just the one that humans in the appropriate society would ordinarily give. So, too, with legal propositions - for a sufficiently aggressive flavor of legal positivism (or a Holmesian (1997) prediction-ist) we might dare argue that legal correctness is just constituted by what a judge would do, and

rulings of prior decisions. However, I'm imposing the artificial constraint on the system (which I shall briefly relax later on), that we are entitled to ignore any hierarchy of authority or weight between cases.

5 This representation is drawn from how machine learning practitioners describe the support vector machine algorithm (see, e.g., Burges 1998, 128).

${ }^{6}$ See James 0'Malley, “Captcha if you can: how you've been training AI for years without realising it," January 12, 2018, at https://www.techradar.com/news/captcha-if-you-canhow-youve-been-training-ai-for-years-without-realising-it . 
just like we can use machine learning to answer that question very reliably for arrangements of pixels, we may be able to do the same for arrangements of legal facts.

We might even make a similar claim from a vaguely Fullerian standpoint. Fuller has argued that the meaning of legal propositions is discoverable only with the help of the practical behavior extant in the social world (Fuller 1976, 58-59; see discussion in Postema 1994, 374-6). We might make a weaker variation on this argument, according to which law can be understood only in aid of some kind of practical applications, be those as manifested in community practices or as manifested in exemplars of their application in the form of judicial opinions. We could then understand the work of DeepVehicle as surfacing a kind of latent legal understanding from the aggregation of practical applications of the statute. ${ }^{7}$

\section{Disagreement and Correctness}

Let us call the story I just sketched out "legal judgment as function approximation." If it adequately represents how jurisprudence works or ought to work, it might seem reasonable to just sack all the judges. Now let's give it some trouble. What I have just described is the skeleton of a model of legal reasoning (of the judicial sub-type, but it's generalizable). It tends to be useful to test our models of reasoning in any kind of domain by examining the plausibility of their approach to one of the most intractable problems of human social cognition, namely, disagreement.

The approach to disagreement in the story I've been telling most closely resembles an error model: if two judges disagree about the correct evaluation of the vehicleness of something in the park, one of them is wrong-one of the judges is doing a better job at accessing the juridical fact of the matter (in a sense loosely corresponding to Dworkin's right answer thesis).

In particular, this story seems to be in tension with any model of legal disagreement according to which it arises as a result of legitimate differences in context or participantsbecause there's a true legal judgment out there, which DeepVehicle is just trying to approximate, we don't have the resources to approve of a diversity of legal judgments rooted in things like the different insights that different judges would bring to the problem. ${ }^{8}$

${ }^{7}$ Fuller, incidentally, would probably reject this use of his work. See discussion in Postema (1994, 379).

${ }^{8}$ We could approve of different judgments resulting from procedural variations in underlying cases-such as the common situation where a party argues some position on appeal which is, according to the other party, waived by the failure to raise it below-by incorporating information about the procedural history of each case into DeepVehicle's input vector. Although this ignores the distinction between substantive and procedural rules, it doesn't seem like an obviously incorrect thing to do: the right answer to a legal question as presented in real-live cases incorporates not just the facts of what happened in the outside world but also the facts of what happened in the judicial process. (This gets 


\section{Dragging the Humans (Kicking and Screaming) Back In?}

One thing that the story above seems to miss is the value of procedural justice, as represented in particular by the capacity of parties to engage in legal argumentation, and, moreover, the capacity for that argumentation to actually make a difference to the result of the case. Suppose DeepVehicle completely replaces our judge. There remains an opportunity for the parties to argue about the facts-was the thing that got brought into the park really on wheels, or was the eyewitness just confused? - but not about the law. ${ }^{9}$ But meaningful legal argumentation seems like an important part of a legitimate system of adjudication.

How could we reintegrate legal argumentation into the story?

First, we could suppose that legal argumentation serves to shape the form of the model. Here is where we can reintroduce many of the complexities that I assumed away at the beginning of this chapter. The task of figuring out what sorts of things go into our input vector, in machine learning, is known as finding a feature representation. Judge Hercules ought to ask herself a few more questions: why should we extract engine presence, number of wheels, and weight from our prior cases and train our model on that? Why not whether there are wings, whether the user has to pedal, and whether the alleged vehicle goes "neigh" or "woof?" This is a different reading of what lawyers do when we distinguish cases, modifying the support vector machine analogy I made above. Imagine the prosecutor has argued that the defendant's Vespa counts as a vehicle in part because of its weight, and the defendant has pointed (either manually or with a machine learning model) to a collection of cases in which the defendant brought things of similar weights into the park and was acquitted. Our prosecutor may continue the dialectic by observing that in all those other cases, the heavy object in question was a St. Bernard, and the St. Bernardiness of the prior non-vehicles is relevant as a means to distinguish the Vespa; that's more or less equivalent to arguing to the data scientist that we ought to include being furry and going "woof" in the input vector and (the prosecutor hopes) watch the half-space of couplehundred-pounds-or-so things separate nicely on that basis.

To be fair to Judge Hercules, some of that stuff can potentially be automated-the lawyer's argument that some heretofore neglected fact was important in deciding the prior case is more-or-less the same thing as the data scientist's (and even social scientist's) use of

more complicated immediately, as the introduction of machine learning into the adjudicative process would change the space of potential lower-court happenings.)

${ }^{9}$ Pasquale (Forthcoming) has the best extant discussion of how the value of legal process informs how we should shape the relationship between human and automated modes of legal judgment. The remainder of this chapter could be seen as my attempt to supplement Pasquale's argument that we ought to focus on automated augmentation rather than replacement of attorney cognitions with a jurisprudential substructure and some additional examples of how such augmentation might be carried out. 
various information-theoretic constructs to automate feature selection (Burnham and Anderson 2004). But some of it can't be automated-we still have to decide on a higherlevel feature representation, for example: we have to determine an underlying ontology for legal representation (like how images are made up out of pixels and texts are made up of words, sentences, or documents-what are legal facts made up out of?). We also have to decide what specific instances of that ontology even enter into our feature selection process: we cannot include every piece of knowledge that we have about the whole universe into our input vector; even an automated-feature selection process relies on preexisting legal judgments like the irrelevance of facts about, for example, what the judge had for breakfast that morning. ${ }^{10}$

Moreover, we also would still have to decide on the functional form of the model and its parameters. ${ }^{11}$ We could try to automatically select some of this using automated search processes (Mendoza et al. 2016) but we would still require a metric for success, as well as a threshold probability to determine the boundary of a classification. ${ }^{12}$ All of those things

${ }_{10}$ Postema $(2014,92-93)$ explains that the description of the underlying facts is itself a subject of argument. In data science terms, we could say that unlike, for example, pixels in an image, the value of each of the elements in an input vector in the law is itself subject to contestation.

${ }^{11}$ Are we doing a deep learning model or a support vector machine? I've shamelessly appealed to features of each in the function approximation story, but they're different algorithms! If a neural network, how many layers? What's the learning rate?

${ }^{12}$ By a metric for success, I mean that in order to train a machine learning model and judge when one is doing a good job of it, data scientists use a variety of metrics to distinguish better over worse models. For intuition, consider that the true positive rate-the percentage of cases in the training data where a judge labels the object as a vehicle and our model also correctly labels it a vehicle-may be very different from the true negative rate (the percentage of cases where the model, like the judge, says "not a vehicle"). Which of these we care more about may differ from setting-to-setting. (For example, minimizing false negatives is much more important in a test for a dangerous, but easily curable with a painless treatment, disease than minimizing false positives, because the harm from one error is much larger than another.) Data scientists have many different metrics, such as accuracy and AUC, to choose from (see Sokolova and Lapalme 2009).

By a threshold probability, I mean that classification models such as DeepVehicle typically don't spit out "yes it's a vehicle" /"no it isn't." Rather they spit out numbers most helpfully interpreted as a estimate of the probability that the observation falls within a given class. It's not obvious that Judge Hercules should rule that an object is a vehicle if DeepVehicle gives a greater than .5 probability of vehicleness-perhaps the correct threshold is .7, for example, with .69 yielding a judicial ruling of non-vehicle. This judgment is going to depend at least in part on what we're trying to achieve with the judgment of vehicleness, as well as on the kinds of considerations that go into other legal probabilistic threshold-setting 
could make up the domain of legal argument and human judgment even in DeepVehicle's world. For example, we could imagine a criminal defense lawyer arguing that the rule of lenity implies that we ought not to conclude that the defendant's machine is a vehicle unless DeepVehicle thinks so with very high probability. ${ }^{13}$

And then there are a whole different set of arguments about how we incorporate the formal rules of authority into this predictive process. Could we, for example, represent the hierarchical authority of different prior courts, the impact of recency, the greater significance of landmark cases, the cutting-off of precedent prior to a statutory amendment, and the like in terms of modeling choices such as weights on different (judicial) observations? Perhaps, but this too would require legal argument, and argument closely integrated with the skills of the data scientist to boot. ${ }^{14}$

More troublingly for DeepVehicle, we could suppose that legal argumentation has a generative rather than an epistemic function. That is, suppose the law is open-textured, in that there is necessary linguistic ambiguity in legal propositions, leaving room for judges to make or modify the law in ways that are not fully captured by the record of prior cases (or even an unavoidable need for such lawmaking activity, whether or not the judge recognizes it as part of what she's doing). Or just suppose that legal argumentation raises matters of policy and of justice that may be legitimately used by the judge in making a determination. Imagine, for example, that DeepVehicle has concluded that anything with 4 or more wheels is always a vehicle, and then, for the first time, someone shows up in the park with a wheelchair. A well-organized legal system ought to give the defendant an opportunity to raise the obvious claim of justice either as an input to the interpretation of the statute or an external side-constraint on it; viewed from a more technocratic perspective we could equally well say that a well-organized legal system ought to have the capacity to take

judgments (like the difference between preponderance of the evidence, clear and convincing evidence, and beyond a reasonable doubt, perhaps?).

${ }^{13}$ Compare Ortner (2016), interestingly using corpus linguistics to apply the rule of lenity.

${ }^{14}$ For example, we might use some kind of model capable of taking into account graph properties of our prior corpus of cases, like citation networks (perhaps using techniques like those described in Hamilton, Ying, and Leskovec 2017). The choice of which graph properties to use would require legal judgments about how those properties affect the authority of precedent. Some of those judgments will be highly controversial. Consider, for example, what such a model would do with a plurality decision of the U.S. Supreme Court. The Court has articulated a rule in Marks v. United States, U.S. 188, 193 (1977): "the holding of the Court may be viewed as that position taken by those Members who concurred in the judgments on the narrowest grounds." While we might be able to imagine ways to represent this in terms of formal properties of cases (such as via concurrence relationships among Justices), it is infamously unclear how to practically apply this rule in ordinary legal analysis (see the discussion in Williams 2017); any particular choice of representation for modeling purposes would be highly debatable. 
account of true novelty, and it's not obvious how DeepVehicle could do that without a real live judge second-guessing its predictions. ${ }^{15}$

Now we're in a whole new story. Let's call it the "permissible disagreement" story, for the points above suggest that, contra the function approximation story, we may be obliged to put up with persistent legal disagreement: two judges with different conceptions of justice and the relative balance of legislative and judicial responsibilities might come to different views on the wheelchair question. ${ }^{16}$ This possibility of reaching disagreement arises from the proposition that preexisting law (or the preexisting right decision, all things considered and on top of the law) is indeterminate, or perhaps we could better say incompletely theorized, and so there isn't any unique legal conclusion for DeepVehicle to discover on that particular set of facts. ${ }^{17}$

15 This seems important from a machine learning perspective too. Otherwise, over time, such a model would just be training on its own predictions. Consider a model trained on $\mathrm{n}$ observations; with the case in which Judge Hercules decides to defer to the model serving as $n+1$. From that case until case $n+$ arbitrarily large $m$, in the absence of additional human judgments, DeepVehicle will be generalizing from the same $\mathrm{n}$ actual human judicial determinations, even if the m intermediate (computer-generated) judgments are fed into the training data as they're created. It's not obvious how DeepVehicle could learn how to handle novel situations in which a human judge would not follow the prior cases, such as our wheelchair example. Judge Hercules might need to assure some continuing human intervention in the ongoing docket, perhaps in the form of some kind of reinforcement learning process amounting to stating rules/her judgments in novel cases as "advice" to a learning model, as in Maclin and Shavlik (1996). (Perhaps we could make use of the $\mathrm{m}$ intermediate judgments in some kind of semi-supervised learning algorithm, like what Hady and Schwenker $(2013,218)$ call "self-training", where we bootstrap training data for far-future predictions on the basis of near-future predictions?) Put differently, we must attend to Postema's $(2014,79-81,86-87)$ point that the introduction of a new decision, as legal rule, into a dynamic system of preexisting decisions and rules, has to perturb that system, and that way permit change over time; moreover, as he suggests, this process is inherently argumentative.

16 If we were doing proper jurisprudence here, this would be a cue for an excursus into the extent to which these different claims of justice could plausibly be incorporated into the notion of a single legally correct answer-but as I am doing improper jurisprudence, we will ignore this altogether. (At any rate, I don't think the distinction is relevant to judging the capacity of DeepVehicle.)

17 In mathematical terms, we might think of a function whose domain isn't known from the start, and where unknown parts of the domain might not map to a single output. 
I will just assert that real-world common-law legal systems partake of both models. ${ }^{18}$ Sometimes, what really matters to us is a correct legal judgment rooted in the context of past judgments - there are cases where there aren't real questions of justice or novelty at stake, we're just trying to crunch a technical legal matter, and disagreement seems problematic. Other times, novel questions of law and justice predominate-consider valueladen Constitutional litigation, where we would expect reasonable judges to disagree all the time. And in many cases we want a little bit of both.

\section{Scoping DeepVehicle: Some Normative Considerations}

Given that we are stuck with judges for the foreseeable future, yet have the inevitable temptation to integrate something like DeepVehicle into their work, it will help us to consider a handful of the many tasks that we, normatively speaking, desire that the law carry out, and think about how DeepVehicle might contribute to or undermine those goals.

\section{Organizing Lives/Predicting Consequences}

One recurrent theme in the rule of law literature (prominent, inter alia, in Hayek and Fuller), is the notion of predictability: individuals are said to be more capable of self-rule, either through or independent of the law, to the extent they are capable of predicting what the law will conclude about their behavior. ${ }^{19}$ Predictability likely also encourages economically beneficial settlement.

There's a sense in which DeepVehicle could be said to do a better job of giving guidance to citizens about the legal outcomes of their planned actions than the current method of adjudication. After all, Judge Hercules can always share her code and data, and assuming she uses good reproducibility practices, citizens could generate, in advance, reliable predictions about how a judge would rule on their proposed behavior, at least to the extent they could generate reliable conclusions about the way in which the facts would be labelled. ${ }^{20}$ In effect, if the judge only rules on the basis of DeepVehicle's predictions, and the

18 Indeed, I'll go further and say (in the spirit of improper jurisprudence) that the usefulness of the two stories I've told so far more-or-less tracks the Hartian distinction between "easy" and "hard" cases. While I'm at it, one more totally undefended allusion to mainline jurisprudence: we could call DeepVehicle a tool to achieve Dworkinian "fit" and say that the continuing employment of Judge Hercules is much more likely to be effective at "justification." Alternatively, we could point to the dynamic tension Waldron $(2008,7-9)$ identifies between the value of determinacy and the value of participatory opportunities.

19 (For discussion of Hayek's view and more on prediction, see Gowder 2016, 68-70; on Fullerian views, see Postema 1994, 370-3.)

20 This rests on a practical assumption about machine learning, namely, that many aspects of machine learning practice are surprisingly easy, and getting easier every day. For example, while training models requires a certain amount of expertise, as of this writing, a 
case is one in which it is clear what input vector to pass to that model, there is a potentially unprecedented degree of certainty to the outcome.

Note, however, that I snuck back in the assumption that it's relatively easy to figure out the correct feature representation of a given case. This may be true for some subcategories of cases, such as those where the relevant legal rule rests entirely on the some closed part of reality, but will obviously be false for many others.

One domain where such advantages might be available would be in evaluating the facial validity of legal documents. Such document evaluation ("does this lease contain any facially void provisions") could be the object of a canonical feature representation that would make it relatively easy for an individual to pass the correct vector into a preexisting model. This is especially true for those legal rules that are effectively a one-way ratchet-if a lease is invalid on its face, e.g. because it has some provision forbidden by a statute, it's typically going to be invalid no matter what extrinsic facts are introduced, while a lease that might look valid on its face could require the introduction of additional facts that still make it invalid-DeepLease could at the very least reduce the range of uncertainty in such cases by allowing some subset of invalid leases to be identified on the spot. ${ }^{21}$

\section{Constraining Power}

A second category of legal value is the law's capacity to constrain official power. The discursive and procedural elements of litigation are central to law's function as constraint, for (as I have argued at some length in Gowder 2016; Gowder 2015) the constraint of power that the rule of law offers inevitably comes through mass action or its threat. The individual capacity to offer legal arguments in such a system is important as a method of communicating claims and outcomes to the rest of the community in order to facilitate broad-based knowledge about official compliance with law.

While this suggests that the value of constraint of power initially might weigh against the acceptability of DeepVehicle, we could imagine some circumstances in which we might be able to use DeepVehicle to help constrain officials in the service of rule of law values. This could be particularly important in criminal law, in which defendants might make plausible

pre-trained model (suitable to generate predictions on new data) can be distributed directly to end-users and can even be executed in an ordinary web browser. (See, e.g., the TensorFlow JavaScript library at https://js.tensorflow.org.)

${ }^{21}$ However, for reasons I discussed in an earlier paper (Gowder 2018) and at more length in a conversation on the "Oral Argument" podcast http://oralargument.org/175, July 21, 2018 , this might actually be bad for egalitarian rule of law values. If we create a world in which the landlord has a computer to catch easy facial blunders in his oppressive leases, so that he can rewrite them without the legal errors but with just as much oppression, we've taken away a relatively cheap way for tenants and their legal advocates to have those leases invalidated and eke out a little bit of justice in a system where the decks are already stacked against them. 
arguments from the rule of lenity and due process/ex post facto principles barring retroactive criminal law for the claim that if DeepVehicle labels their behavior as noncriminal, any new rule labelling it as criminal can operate prospectively only.

DeepVehicle might also be useful as an external check on government claims of discretion rooted in legal indeterminacy. For example, in the United States, the qualified immunity defense is available under 42 U.S.C. 1983 to state officials who violate civil rights if the law prohibiting their conduct is not "clearly established"; this doctrine has been criticized for requiring such a high degree of certainty in the wrongfulness of official conduct that it effectively denies the victims of (for example) egregious police abuse any remedy (Reinhardt 2015, 1244-50; Carbado 2016, 1519-22; Feldman 2017). Potentially, something like DeepVehicle (Call it DeepBeat? My, ahem, deepest apologies to techno musicians.) could be used as a floor on clearly established doctrine-perhaps if a preexisting model predicts that an official's behavior is illegal with sufficiently high confidence, that misbehavior should count as clearly established. Of course, this idea could also be abused by officials in the other direction, to claim that the illegality of some blatantly abusive behavior isn't clearly established because the model doesn't happen to pick it out. ${ }^{22}$

\section{Output of Democratic Sovereignty}

One of the tasks that we ask of law is that it transmit commands from a democratic sovereign to its members and officials, although the precise details of when law is properly attributable to a popular sovereign is a matter of longstanding debate. At least sometimes, we probably have some pro tanto reason, for the purposes of implementing the democratic or legislative will, to interpret law consistent with its legislative intent or popular understanding. Again, there's endless controversy here, but we also have a regular judicial

22 That being said, if "clearly established" is outrageously narrow, there's probably more room for DeepBeat to expand rather than contract the set of behaviors subject to liability, at least in the short-term. Essentially the question comes down to how much similarity to preexisting facts is required under current doctrine versus under DeepBeat: if qualified immunity doctrine as it currently stands requires the misconduct to essentially be identical to the facts of some prior case ("your honor, I know the prior cases said the police can't hit cuffed prisoners with a stick, but my client hit the prisoner with a brick-not clearly established!"), then a machine learning model that can generalize just a little bit from those facts would be an improvement. Analogously, one of the core problems in machine learning is the "bias-variance tradeoff" (James et al. 2013, 33 et. seq.), essentially the problem that you always have to choose between a relatively simple model that can generalize to unseen data but doesn't take into account all the information we have and a relatively complex model that generalizes less well ("overfits") but is more capable of representing the data we already have. In those terms, the criticism of qualified immunity doctrine is that it overfits the narrow facts of particular prior cases, and ought to be swapped out with something (DeepBeat or a less terrible legal rule) that generalizes better. 
practice of interpreting statutes in accordance with some notion of legislative intent, so I shall simply rest on that observation and proceed. ${ }^{23}$

We might perhaps put DeepVehicle to work in this context as a tool to connect statutory interpretation and legislative or popular cognitions. Consider the case of a legislature looking to exercise more fine-grained control over how the courts interpret its statutes. As it presently stands, perhaps legislators can make use of things like canons of statutory interpretation to predict and shape how courts will interpret their enactments, but their present ability to use those tools is uncertain at best (Gluck and Bressman 2013). DeepVehicle might enhance shared knowledge between legislatures and courts about how particular provisions in statutory text would be interpreted. If we had, for example, a dataset sufficient to generate predictions about how courts would interpret different formulations of the definitions section in our "no vehicles in the park" statute, we could use it to coordinate judicial and legislative interpretations of that section-legislators could choose the language most consistent with their intent, confident that courts using the same machine learning model would interpret the statute accordingly. ${ }^{24}$

Alternatively, we might conceive of DeepVehicle as a tool for creating statutory intent. Hurd (1990) has argued that legislative enactments cannot count as communication in a Griceian sense because that account of communication requires both a speaker's intentnotoriously difficult to determine in the case of a legislature-as well as an audience that can receive that intent-notoriously difficult to achieve in the case of mass audiences who at best learn the meaning of statutes through intermediate communicators (the press, their lawyers). Perhaps DeepVehicle could allow us to attribute an intention to the legislature to pick out the cases covered by the predictions the model generates from their language, and at least have some hope that ordinary citizens would have access to the tools to "listen" to

${ }^{23}$ Here permit me to resist being read to make any particular commitments in democratic theory. As it turns out, I am currently working on a conception of popular sovereignty (in a different paper) that denies the claim that citizen cognitions must have some special power over individual day-to-day political outcomes, which makes everything here a bit troublesome. Other people seem to think it matters, the observations in this section may be useful to them.

${ }^{24}$ One objection: if the legislature could think of the cases that they wanted to be sure counted as vehicles, they could just write them directly in the text; the problem with statutory interpretation is what to do with the cases the legislators couldn't dream up. This problem can be made to dissolve with a little bit more effort from the computational side: neural networks can generate novel data similar to their training sets-like Chinese poetry (Zhang and Lapata 2014)!- so DeepVehicle could generate imaginary arrangements of facts that are/are not predicted to count as vehicles so that the legislators may see the hidden implications of their text and correct for any that they dislike. In software engineering terms, we can think of this as a kind of intelligent fuzzing (Oehlert 2005), where we use machine learning to generate hypothetical cases for our proposed statute and see if anything goes wrong. 
that communication by repeating the prediction (this obviously requires much more theoretical development).

That being said, DeepVehicle would also require some limitations in order to protect democratic sovereignty, particularly to the extent that sovereignty is understood to consist, in part, in a discursive relationship with legal decision makers. ${ }^{25}$ On such a conception of democratic sovereignty, the people must be understood to have some capacity to criticize and (ultimately) change legal conclusions-and thus, in computational terms, to invalidate the labels in the existing datasets. The points I made above about the necessary capacity for judges to make new law, in connection with the wheelchair hypothetical, will apply with equal force to the capacity of legislatures and mass publics to do so. Without more fully developing an account of how exactly the people do make law and engage in discourse with their legal systems I cannot fill out here precisely what the consequent limitations on DeepVehicle would be.

\section{Conclusion: Legal Cognition Ain't Just Cognition}

In the context of all these points, it may be best to see our philosophical thought experiment of what would happen if we inserted machine learning into adjudication as pointing to the irreducibly discursive nature of legal judgment. Thus, I assert the following propositions.

There might actually be a use for DeepVehicle. Machine learning could be juridically useful in informing litigants and courts about generalizations from prior decisions and facilitating higher inter-case consistency, particularly in those types of cases that appear with high frequency and that are characterized by well-established factual representations in their domains. Document-based practices may be most amenable to DeepVehicle, such as administrative law in contexts such as securities and patents, and even Social Security disability claims (to the extent medical records are predictive of outcomes) as well as leases, some kinds of insurance claims, and the like.

We'd have to lawyer it like usual. The shape of any model would continue to be subject to ordinary legal process. We would expect lawyers to debate, and judges to decide, things like the success metrics, classification thresholds, and underlying ontology as well as the features represented in the input vector for any model. Indeed, the decisions about many of these inputs to the machine learning process bear a striking isomorphism to ordinary modes of lawyerly reasoning, with the most salient example being the correspondence between the lawyer's practice of identifying relevant facts to distinguish or follow prior cases and the data scientist's task of feature selection. Judge Hercules keeps her job.

The uses of and weight placed on any such models would have to be subject to continuing scrutiny and would also vary by area. For an obvious example relating to one of the areas noted above: it would be dangerous and almost certainly unjust to deny

25 See, e.g. Dworkin (1997, 343-7); Gowder (2014b). 
Social Security disability claims solely on the basis of a machine learning fit from medical records, in light of, among many other reasons, disparities in access to treatment. Similarly, in the criminal law, we might hope that courts interested in basic legal values would choose to use machine learning adjudicative tools in a way heavily favoring defendants-as noted above, perhaps with results that are binding when defendant-favoring but not when prosecutor-favoring. Ordinary principles of law and justice such as the requirement that ambiguities be resolved in favor of criminal defendants would need to be translated into machine learning terms.

\section{Common law methods are well-suited to manage the incorporation of machine} learning into law. The common law might be described as the art and science of muddling through in the context of novelty: evolving robust legal rules in the face of novel facts, via judges doing the best they can on a case-by-case basis, and drawing liberally from adjacent cognitive domains (neighboring doctrines, neighboring intellectual disciplines, and neighboring frameworks for normative decision in the form of social norms). This is precisely what would be needed to handle the use of machine learning in legal adjudication: we would need to develop meta-rules governing the use of such tools. ${ }^{26}$ Thus, Judge Hercules may be wise to borrow rules from the existing law covering other areas of adjudication. For example, we might imagine building on existing rules of precedent to develop rules as to which cases are included in the training data and the weights to be placed on them, depending on jurisdiction, hierarchical level of the issuing court, date, and the like. We might borrow from evidence and civil procedure to figure out what weight to give to the models themselves-drawing analogies between DeepVehicle and, for example, an expert witness, a special master, or a lower court subject to a standard of review on appeal. All of these meta-level legal judgments would likely have to evolve in pragmatic common-law fashion, incrementally incorporating prior experience into ongoing judicial experimentation. ${ }^{27}$

The legal academy would need to step up. The story of this chapter has not just been a thought experiment, but also an implicit exercise in speculative disciplinary sociology: it makes some claims about what would have to happen if (when?) the core of the discipline of law is invaded by the tools and methods of the discipline of artificial intelligence. We have seen this before, in the growth of law and economics, and at a very high altitude, this paper borrows from the experience of law and economics to imagine how the development of law and artificial intelligence might happen. Notably, law and economics was driven by a

${ }^{26}$ We would also need to finally carry out a long-overdue task: integrate the professional insights and experiences of lawyers with those of ethicists and computer scientists in the burgeoning literature on algorithmic bias (see generally Garcia 2016).

${ }^{27}$ Contemplating those meta-rules reveals an irony at the heart of DeepVehicle. Judge Hercules considered relying on it in order to promote consistency and certainty, but as the second-order rules for making use of DeepVehicle become more complicated, they in turn may become difficult to apply, and the very reasons for DeepVehicle's introduction may begin to slip away. 
narrow but influential cadre of intellectual entrepreneurs in the academy and on the bench. In artificial intelligence, the scholarly cadre is already moving, but it won't get really interesting until (unless?) the judicial cadre forms (as Calabresi, Posner, and the like served the Herculean role in law and economics). ${ }^{28}$ If Hercules the Judicial Data Scientist does show up, this disciplinary invasion will have to be integrated within the intellectual framework of law in order to achieve the benefits of artificial intelligence without the sacrifice of core legal values. On the practical and instructional side, that integration would require a broader training of future lawyers and judges in the methods with which they will be confronted: as every law student now learns at least the rudiments of ideas like the difference between property rules and liability rules, law schools may have to incorporate the epistemology of machine learning into the curriculum in order to prepare the bench and bar for what it may face. ${ }^{29}$ On the scholarly side, legal academics may be called upon both to manage the interdisciplinary interface between machine learning methods developed in the academy (and in industry) and legal methods, and to serve their traditional role of systematizing and rationalizing the case-by-case experimentation of judges.

Ultimately, reflecting on machine learning as an element of adjudication should lead us to more vividly appreciate the fact that legal cognition isn't just cognition. It serves social functions and is embedded into networks of relationships that mediate hierarchical social categories (cf. Gowder 2018) as well as procedural systems that encapsulate important judgments of political entitlement. Replicating the cognitive operations of legal professionals on either side of the bench is the easy part-that's just engineering-but mapping computational cognition to their social roles is far more difficult and interesting.

28 The sociological predictions of this chapter also mirror our experience with law and economics: despite the ambitions of its founders, economic methods did not take over the field of law; they never did manage to swap out the traditions of the common law for, e.g., Coasean transaction cost economics, although those insights have been put to fruitful use across the discipline.

29 This also means that law students will have to be taught to abandon their traditional terror of math. The undersigned is currently preparing a fully open-source course, entitled "Introduction to Quantitative and Computational Legal Reasoning," which is aimed at making some progress along these lines (readers may access or contribute to the materials for it at https://sociologicalgobbledygook.com); many of the contributors to this volume have conducted similar experiments. I very much hope that the legal academy develops a distinctive mathematical/statistical pedagogy and community of knowledge around such teaching in the near future, one that draws instructional lessons from the experience of our colleagues in computer science and statistics (such as the method of instruction by problem sets) without picking up their vices (such as the shamelessly lazy method of lecturing by reading proofs off a slide). 


\section{References}

Aletras, Nikolaos, Dimitrios Tsarapatsanis, Daniel Preoţiuc-Pietro \& Vasileios Lampos. 2016. "Predicting judicial decisions of the European Court of Human Rights: a Natural Language Processing perspective." PeerJ Computer Science 2:e93.

Burges, Christopher J. C. 1998. "A Tutorial on Support Vector Machines for Pattern Recognition." Data Mining and Knowledge Discovery 2 (2): 121-67.

Burnham, Kenneth P., and David R. Anderson. 2004. "Multimodel Inference: Understanding AIC and BIC in Model Selection." Sociological Methods \& Research 33 (2): 261-304.

Carbado, Devon W. 2016. "Blue-on-Black Violence: A Provisional Model of Some of the Causes." Georgetown Law Journal 104 (6): 1479-1530.

Dworkin, Ronald. 1986. Law's Empire. Cambridge, MA: Harvard University Press.

- - - 1997. Freedom's Law: The Moral Reading of the American Constitution. Cambridge, MA: Harvard University Press.

Feldman, Leonard C. 2017. "Police Violence and the Legal Temporalities of Immunity." Theory \& Event 20 (2): 329-50.

Fuller, Lon. 1976. Anatomy of the Law. Westport, Conn.: Greenwood Press.

Garcia, Megan. 2016. "Racist in the Machine: The Disturbing Implications of Algorithmic Bias.” World Policy Journal 33 (4). Duke University Press: 111-17.

Gluck, Abbe R, and Lisa Schultz Bressman. 2013. "Statutory Interpretation from the Insidean Empirical Study of Congressional Drafting, Delegation, and the Canons: Part I." Stanford Law Review 65 (5). HeinOnline: 901-1025.

Gowder, Paul. 2014a. "Equal Law in an Unequal World." Iowa Law Review 99 (3): 1021-81.

_-_. 2014b. "The Countermajoritarian Complaint." Transnational Law and Contemporary Problems 23: 7-33.

- - 2015. "What the Laws Demand of Socrates—and of Us." The Monist 98 (4): 360-74.

-_- 2016. The Rule of Law in the Real World. New York: Cambridge University Press.

-_- 2018. "Transformative Legal Technology and the Rule of Law." University of Toronto Law Journal 68 (S1): 82-105.

Hady, Mohamed Farouk Abdel, and Friedhelm Schwenker. 2013. "Semi-Supervised Learning." In Handbook on Neural Information Processing, edited by Monica Bianchini, Marco Maggini, and Lakhmi Jain C, 215-39. Intelligent Systems Reference Library. Springer, Berlin, Heidelberg.

Hamilton, William L., Rex Ying, and Jure Leskovec. 2017. "Representation Learning on Graphs: Methods and Applications." IEEE Data Engineering 40 (3): 52-74. 
Hart, H.L.A. 1958. "Positivism and the Separation of Law and Morals." Harvard Law Review 71 (4): 593-629.

Holmes, Oliver Wendell. 1997 (reprint). "The Path of the Law.” Harvard Law Review 110 (5): 991-1009.

Hurd, Heidi M. 1990. “Sovereignty in Silence.” The Yale Law Journal 99 (5): 945-1028.

James, Gareth, Daniela Witten, Trevor Hastie, and Robert Tibshirani. 2013. An Introduction to Statistical Learning : With Applications in R. 7th ed. New York: Springer.

Katz, Daniel M., Michael J. Bommarito \& Josh Blackman. 2017. "A General Approach for Predicting the Behavior of the Supreme Court of the United States." PLoS ONE 12 (4): e0174698.

Lin, Henry W., Max Tegmark, and David Rolnick. 2017. “Why Does Deep and Cheap Learning Work so Well?” Journal of Statistical Physics 168 (6): 1223-47.

Maclin, Richard, and Jude W. Shavlik. 1996. "Creating Advice-Taking Reinforcement Learners." Machine Learning 22 (1-3): 251-81.

Mendoza, Hector, Aaron Klein, Matthias Feurer, Jost Tobias Springenberg, and Frank Hutter. 2016. “Towards Automatically-Tuned Neural Networks." In Workshop on Automatic Machine Learning, 58-65.

Oehlert, P. 2005. "Violating Assumptions with Fuzzing." IEEE Security Privacy 3 (2): 58-62.

Ortner, Daniel. 2016. "The Merciful Corpus: The Rule of Lenity, Ambiguity and Corpus Linguistics." Boston University Public Interest Law Journal 25 (1): 101-42.

Pasquale, Frank A. Forthcoming. "A Rule of Persons, Not Machines: The Limits of Legal Automation." George Washington Law Review.

Pasquale, Frank, and Glyn Cashwell. 2018. "Prediction, Persuasion, and the Jurisprudence of Behaviourism.” University of Toronto Law Journal 68 (1 supplement): 63-81.

Postema, Gerald J. 1994. “Implicit Law.” Law and Philosophy 13 (3): 361-87.

——— 2014. "Law's System: The Necessity of System in Common Law." New Zealand Law Review 2014 (1): 69-105.

Reinhardt, Stephen R. 2015. "The Demise of Habeas Corpus and the Rise of Qualified Immunity: The Court's Ever Increasing Limitations on the Development and Enforcement of Constitutional Rights and Some Particularly Unfortunate Consequences Essay." Michigan Law Review 113 (7): 1219-54.

Sokolova, Marina, and Guy Lapalme. 2009. "A Systematic Analysis of Performance Measures for Classification Tasks." Information Processing \& Management 45 (4): 427-37. 
Waldron, Jeremy. 2004. "The Rule of Law as a Theater of Debate." In Dworkin and His Critics: With Replies by Dworkin, edited by Justine Burley, 319-36. Malden, MA: Wiley Online Library.

- - 2008. "The Concept and the Rule of Law." Georgia Law Review 43 (1): 1-61.

Williams, Ryan C. 2017. “Questioning Marks: Plurality Decisions and Precedential Constraint." Stanford Law Review 69 (3): 795-865.

Zhang, Xingxing, and Mirella Lapata. 2014. "Chinese Poetry Generation with Recurrent Neural Networks." In Proceedings of the 2014 Conference on Empirical Methods in Natural Language Processing (EMNLP), 670-80. 